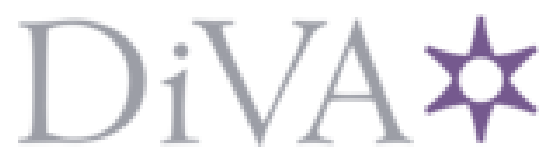

http://www.diva-portal.org

\title{
Postprint
}

This is the accepted version of a paper presented at European Microwave Conference 2017.

Citation for the original published paper:

Dancila, D., Beuerle, B., Shah, U., Rydberg, A., Oberhammer, J. (2017)

Micromachined Cavity Resonator Sensors for on Chip Material Characterisation in the 220-330

GHz band.

In: Proceedings of the 47th European Microwave Conference, Nuremberg, October 8-13, 2017

N.B. When citing this work, cite the original published paper.

Permanent link to this version:

http://urn.kb.se/resolve?urn=urn:nbn:se:kth:diva-216957 


\section{Micromachined Cavity Resonator Sensors for on Chip Material Characterisation in the 220-330 GHz band}

\author{
Dragos Dancila, Anders Rydberg \\ Division of Solid-State Electronics, Uppsala University \\ Uppsala, Sweden \\ e-mail: dragos.dancila@angstrom.uu.se
}

\author{
Bernhard Beuerle, Umer Shah, Joachim Oberhammer \\ Micro and Nanosystems, KTH Royal Institute of Technology \\ Stockholm, Sweden
}

\begin{abstract}
An on-chip sensor for measuring the complex relative permittivity in the band $220-330 \mathrm{GHz}$ has been designed. The sensor is based on a micromachined cavity resonator provided with an opening in the top side of a hollow waveguide for sensing. The waveguide is realized by microfabrication in a silicon wafer and is gold metallized. The extraction of the materials characteristics is performed using cavity resonator techniques. Preliminary measurements of several dielectric materials are performed, demonstrating the potential of the sensor and methodology.
\end{abstract}

Keywords—cavity resonators; sensors; WR-3; microfabrication

\section{INTRODUCTION}

The characterization of dielectric properties in the band $220-330 \mathrm{GHz}$ is necessary for different applications such as dielectric heating, remote sensing and molecular detection [1]. At these frequencies, the dielectric permittivity of water in a hydration shell is different from that of bulk water due to the mutual coupling between biomolecules and the hydration shell. As the biomolecular processes change the hydration shell, so does its dielectric properties. The measurement of the dielectric properties can lead to a label-free, immobilization-free, realtime, liquid-phase detection technique [2]. It is also important to derive in an easy way the dielectric characteristics of high frequencies substrates, lenses and antenna radomes for the ongoing development of communication systems at millimeter waves [3]. Different methods for measuring the complex relative permittivity have been adapted for such high frequencies such as free-space methods using a vector network analyzer (VNA) and time-domain spectroscopy (TDS) [4]. However only a few are capable of operating with liquids: single wire transmission line [5], substrate integrated waveguide (SIW) loaded with a capillary tube [6] and planar transmission lines but these are quite lossy which limits the interaction length. Waveguides offer lower losses solutions, yet better accuracy and precision can be obtained with a low loss (high Q-value) resonator, since amplitude of the EM-fields are higher at resonance, which improves the detection sensitivity. Typically, cavity resonators are used for the dielectric characterization of materials and could also be used for measurements of the sheet resistance and conductivity of thin films [7]. In this paper, we developed several cavity resonators operating in the band $220-330 \mathrm{GHz}$ which could be used for dielectric characterization, adapting the methodology devised for lower frequencies resonators. Differently from having the dielectric inserted into the cavity resonator it will instead be in close proximity and evanescently coupled to the cavity resonator. By using the proximity coupling the Q-value of the cavity can be kept high. A number of micromachined sensors fabricated in a novel silicon technology (to be presented elsewhere) has been fabricated with a Q-value of above 600 to evaluate this technique at WR3-band.

\section{DESIGN}

\section{A. Cavity resonator}

For an empty, air filled resonant cavity, the resonant frequency is found using the

$$
f_{101}=\frac{c}{2 \pi \sqrt{\varepsilon_{r} \mu_{r}}} \sqrt{\left(\frac{\pi}{a}\right)^{2}+\left(\frac{\pi}{d}\right)^{2}}
$$

where $a$ and $d$ are respectively the width and length of the cavity resonator, $\varepsilon_{r}$ and $\mu_{r}$ are the relative permittivity and permeability of the filling material in the cavity. The cavity resonator with its $\mathrm{E}$ field and the non-radiating slot evanescently coupling with the material under test (MUT) is shown in Figure 1.

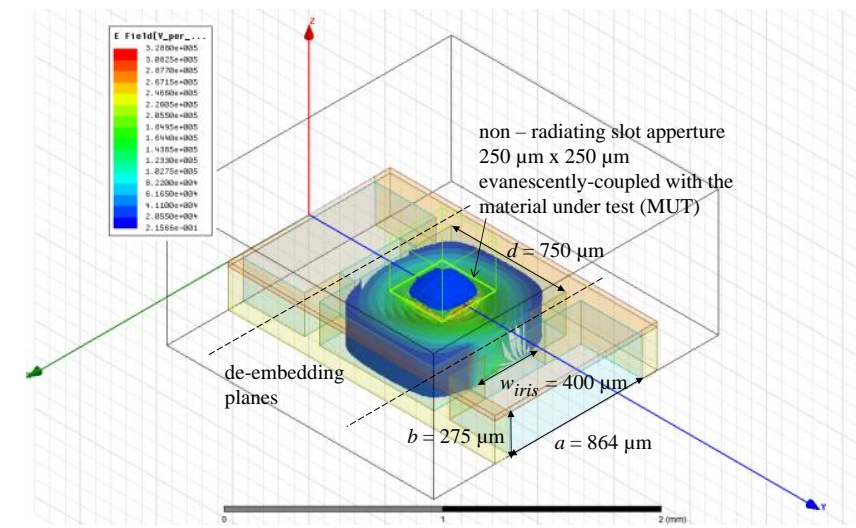

Figure 1: Cavity resonator sensor with E field and nonradiating slot in contact with the material under test.

The width $\mathrm{a}=864 \mu \mathrm{m}$ of the rectangular cavities is fixed by the WR-3 waveguide dimensions and the design parameter is the length $d$ which is used to fix the resonant frequency. 
The height of the waveguide is also fixed by the technology, $b=285 \mu \mathrm{m}$. A two port cavity resonator filter was designed at 240 and $260 \mathrm{GHz}$. Considering the coupling coefficients from using an inductive irises, it was found that an opening width of $400 \mu \mathrm{m}$ ensures a critical coupling.

\section{B. Cavity resonator sensor}

The sensor is based on a cavity resonator provided with an aperture in the top waveguide surface which is evanescentlycoupled to a dielectric material under test. The top wall of the cavity resonator has a thickness of $30 \mu \mathrm{m}$ and dielectrics are place on the opening of $250 \mu \mathrm{m}$ by $250 \mu \mathrm{m}$. The resonant frequency is affected by any dielectric material placed on top of the opening. The coupling to the dielectric material is merely capacitive, as the opening is located at the maximum of the $E$ field. The dimensions of the cavity sensor and coupling irises are optimized using HFSS. Phase response of the cavity resonator sensor for different permittivities varied from 1 to 12 is presented Figure 2.

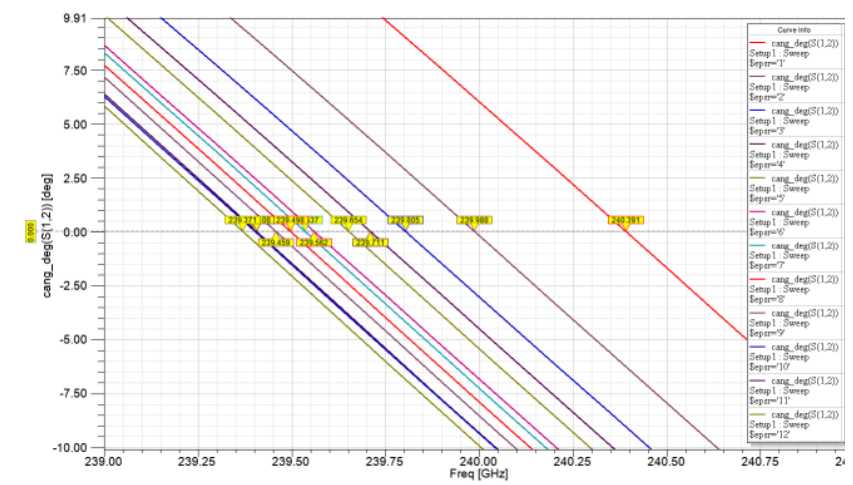

Figure 2: Phase response of the cavity resonator sensor for different permittivities varied from 1 to 12 of the MUT.

\section{Qfactor extraction}

The unloaded-Q factor of the cavity resonator was estimated by measuring the loaded $Q$ factor and coupling coefficients using the following expression:

$$
Q_{0}=Q_{L}\left(1+k_{1}+k_{2}\right)
$$

where the coupling coefficients $k_{1}$ and $k_{2}$ are obtained applying the reflection type $\mathrm{Q}$ factor extraction to a transmission cavity, from the measured coupling coefficients, following a methodology described in [8]. This method is more accurate when the coupling becomes strong, which is the case for the critically coupled filters designed.

\section{Dielectric permittivity extraction}

The cavity perturbation theory provides a way to analyze the impact on the on the complex frequency of a material inserted into the cavity resonator, as follows [9-10]:

$$
\frac{d \omega}{\omega}+\frac{j}{2}\left[\frac{1}{Q_{s}}-\frac{1}{Q_{o}}\right] \approx-\frac{\left(\varepsilon_{r}-1\right)}{2} \frac{\int_{V_{s}} E . E_{0}^{*} d V}{\int_{V_{c}}\left|E_{0}\right|^{2} d V}
$$

Separating real and imaginary parts, results in:

$$
\begin{aligned}
\epsilon^{\prime} & =\frac{1}{2}\left(\frac{f_{o}}{f_{s}}-1\right) \frac{V_{c}}{K V_{s}}-1 \\
\epsilon^{\prime \prime} & =\frac{1}{4}\left(\frac{1}{Q_{s}}-\frac{1}{Q_{o}}\right)^{-1} \frac{V_{c}}{K V_{s}}
\end{aligned}
$$

where $\varepsilon_{r}=\epsilon_{r}^{\prime}-j \epsilon_{r}^{\prime \prime}$ is the relative complex permittivity of the sample, $V_{s}$ and $V_{c}$ are respectively the volumes of the sample and cavity resonator, respectively and $K$ is the coupling correction factor.

\section{E. Coupling correction factor}

The coupling correction factor is taken as the ratio between the resonant frequency obtained using HFSS simulations and the theoretical resonant frequency obtained using the cavity perturbation theory. In the latest, the total volume considered as the non-perturbed volume is cavity resonator volume in addition of the perturbation volume of $250 \mu \mathrm{m}^{3}$. The coupling correction factor is inversely proportional to the relative permittivity of the MUT, as can be seen in Figure 3.

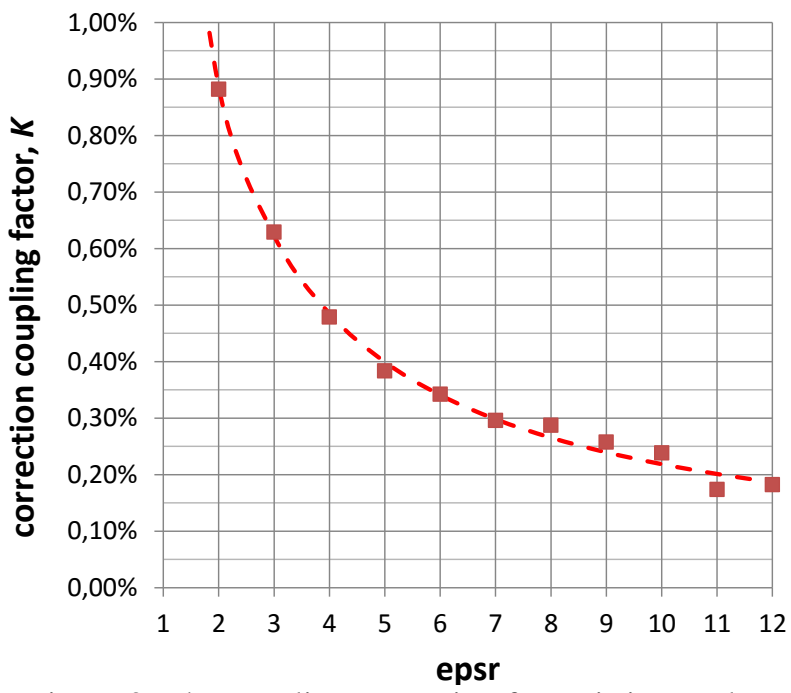

Figure 3: The coupling correction factor is inversely proportional to the relative permittivity of the MUT.

The coupling correction factor is reduced for higher permittivities due to less coupling of EM-fields, see Figure 4.

a)

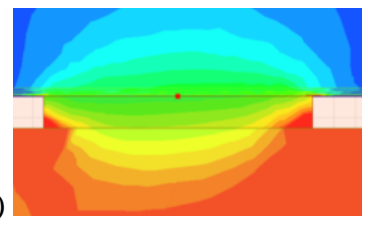

b)

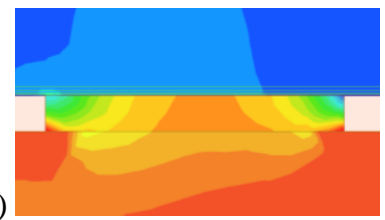

Figure 4: Evanescent coupling and E field at the location of the sensing slot for two relative permittivities of (a) $\varepsilon_{r}=1$ and (b) $\varepsilon_{r}=12$.

This correction coupling factor will be used to extract the permittivities from the measurements. 


\section{MEASUREMENTS}

Measurements were conducted using an R\&S Vector Network Analyzer with millimeter-wave measurement heads in the band 220-330 GHz.

Measurements are performed on different cavity resonators filters provided with an opening with dimensions $(250 \times 250$ $\mu \mathrm{m})$ in the top metallized waveguide), see Figure 1. Different dielectric materials are placed on top of the sensing area and the $\mathrm{S}$ parameters are measured. Materials measured are:

- $600 \mu \mathrm{m}$ thick high-resistivity silicon $(>4000 \Omega \mathrm{cm}$, $\varepsilon_{\mathrm{r}}=11.6, \tan \delta$ of $6 \times 10-4$ measured at $\left.100 \mathrm{GHz}\right)$

- $127 \mu \mathrm{m}$ thick RO3003 high frequency substrate $\left(\varepsilon_{\mathrm{r}}=3\right.$, $\tan \delta$ of $1 \times 10-3$ measured at $10 \mathrm{GHz}$ )

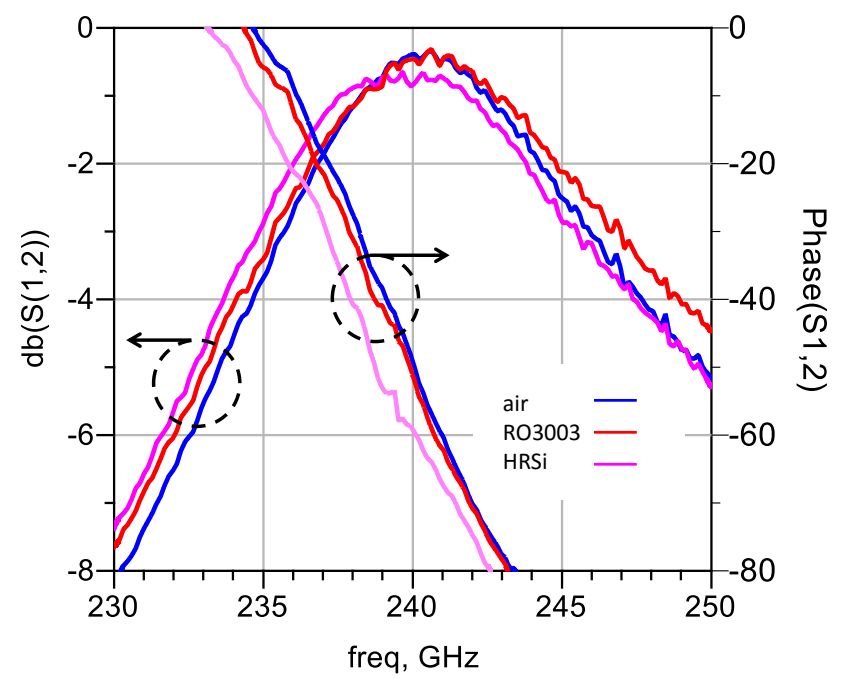

Figure 5: Cavity resonator sensor at $240 \mathrm{GHz}$ measuring three different MUTs: air, RO3003 and HRSi.

In Figure 6 is shown a comparison between measurements and simulation where the trend between resonance frequency and permittivity are quite similar. The frequencies, FL extracted from the measurements correspond to the frequencies at a constant phase of $-60^{\circ}$.

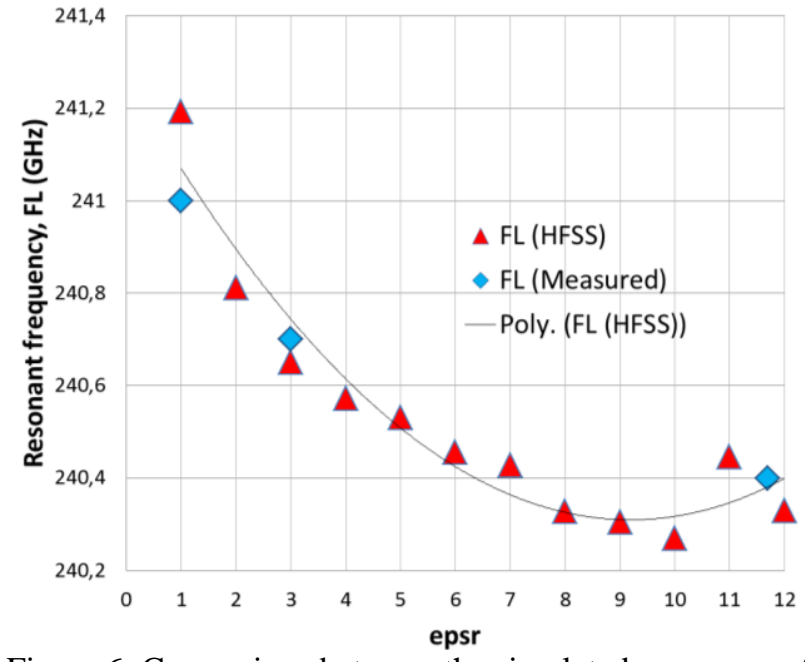

Figure 6: Comparison between the simulated responses of the sensor for different permittivities varied from 1 to 12 and three measured MUTs samples.

The frequency shift is reduced for higher permittivities, due to a reduction of coupling of the EM-fields due to the higher permittivity of the MUT, which is represented by a reduced correction coupling factor, see Figure 3.

A number of cavities with different dimensions and Qvalues will be presented at the conference.

\section{CONCLUSION}

A sensor applicable for $\mathrm{THz}$ applications has been evaluated at the WR-3 band for near proximity coupling.

\section{ACKNOWLEDGMENTS}

The research was financed by the Swedish Foundation for Strategic Research within the MEMS terahertz systems project. Prof. Darko Kajfez is acknowledged for fruitful discussions on the $\mathrm{Q}$ factor extraction of cavity resonators.

\section{REFERENCES}

[1] A. H. Sklavounos and N. S. Barker, "Liquid-Permittivity Measurements Using a Rigorously Modeled Overmoded Cavity Resonator," in IEEE Transactions on Microwave Theory and Techniques, vol. 62, no. 6, pp. 1363-1372, June 2014. doi: 10.1109/TMTT.2014.2321348

[2] T. Shimizu, S. Kojima and Y. Kogami, "Accurate Evaluation Technique of Complex Permittivity for Low-Permittivity Dielectric Films Using a Cavity Resonator Method in 60-GHz Band," in IEEE Transactions on Microwave Theory and Techniques, vol. 63, no. 1, pp. 279-286, Jan. 2015. doi: 10.1109/TMTT.2014.2375830

[3] T. Tosaka, K. Fujii, K. Fukunaga and A. Kasamatsu, "Development of Complex Relative Permittivity Measurement System Based on FreeSpace in 220-330-GHz Range," in IEEE Transactions on Terahertz Science and Technology, vol. 5, no. 1, pp. 102-109, Jan. 2015. doi: 10.1109/TTHZ.2014.2362013

[4] V. Matvejev, Y. Zhang and J. Stiens, "High performance integrated terahertz sensor for detection of biomolecular processes in solution," in IET Microwaves, Antennas \& Propagation, vol. 8, no. 6, pp. 394-400, April 24 2014. doi: 10.1049/iet-map.2013.0366

[5] Laurette, S., Treizebre, A., Elagli, A., et al.: 'Highly sensitive terahertz spectroscopy in microsystem', RSC Adv., 2012, 2, pp. 10064-10071 
[6] Matvejev, V., de Tandt, C., Ranson, W., Stiens, J., Vounckx, R., Mangelings, D.: 'Integrated waveguide structure for highly sensitive

$\mathrm{THz}$ spectroscopy of nano-liter liquids in capillary tubes', Prog. Electromagn. Res., 2011, 121, pp. 89-101

[7] J. Krupka and W. Strupinski, Measurements of the sheet resistance and conductivity of thin epitaxial graphene and SiC films, Applied Physics Letters, vol. 96, 2010 .
[8] D. Kajfez, "Reflection-type Q factor measurement of transmission-type cavities," Proc. of Asia-Pacific Microwave Conf. pp. 449 - 452, 2000. DOI: 10.1109/APMC.2000.925855

[9] D. M. Pozar, Microwave Engineering. Addison-Wesley, 1990.

[10] K. T. Mathew, "Perturbation Theory," Encyclopedia of RF and Microwave Eng., Vol. 4, 3725-3735, Wiley-Interscience, USA, 2005. 